\title{
Counter-Example Guided Predicate Abstraction of Hybrid Systems ${ }^{\star}$
}

\author{
Rajeev Alur ${ }^{1}$, Thao Dang ${ }^{2}$, and Franjo Ivančić ${ }^{1}$ \\ 1 University of Pennsylvania \\ 2 VERIMAG
}

\begin{abstract}
Predicate abstraction has emerged to be a powerful technique for extracting finite-state models from infinite-state systems, and has been recently shown to enhance the effectiveness of the reachability computation techniques for hybrid systems. Given a hybrid system with linear dynamics and a set of linear predicates, the verifier performs an on-the-fly search of the finite discrete quotient whose states correspond to the truth assignments to the input predicates. The success of this approach depends on the choice of the predicates used for abstraction. In this paper, we focus on identifying these predicates automatically by analyzing spurious counter-examples generated by the search in the abstract state-space. We present the basic techniques for discovering new predicates that will rule out closely related spurious counter-examples, optimizations of these techniques, implementation of these in the verification tool, and case studies demonstrating the promise of the approach.
\end{abstract}

\section{Introduction}

Inspired by the success of model checking in hardware verification and protocol analysis 12 20], there has been increasing research on developing tools for automated verification of hybrid (mixed discrete-continuous) models of embedded controllers 146 6161922. Model checking requires the computation of the set of reachable states of a model, and in presence of continuous dynamics, this is typically undecidable. Consequently, contemporary tools for model checking of hybrid systems, such as CheckMate[9] and d/dt [6], approximate the set of reachable states by polyhedra. It has been shown that effectiveness of the reachability computation for hybrid systems can be enhanced using predicate abstraction [3. Predicate abstraction is a powerful technique for extracting finite-state models from complex, potentially infinite-state, discrete systems (see, for instance, [14 23]), and tools such as Bandera [13], SLAM [7], and Feaver [21] have used it for analysis of $\mathrm{C}$ or Java programs. The input to our verification tool consists of the concrete system modeled by a hybrid automaton, the safety property to be verified, and a finite set of predicates over system variables to

\footnotetext{
* This research was supported in part by ARO URI award DAAD19-01-1-0473, DARPA Mobies award F33615-00-C-1707, NSF award ITR/SY 0121431, and European IST project CC (Computation and Control).
} 
be used for abstraction. For the sake of efficiency, we require that all invariants, guards, and discrete updates of the hybrid automaton are specified by linear expressions, the continuous dynamics is linear, possibly with bounded input, and the property as well as the abstraction predicates are linear. An abstract state is a valid combination of truth values to the predicates, and thus, corresponds to a polyhedral set of the concrete state-space. The verifier performs an on-the-fly search of the abstract system by symbolic manipulation of polyhedra.

The core of the verifier is the computation of the transitions between abstract states that capture both discrete and continuous dynamics of the original system. Computing discrete successors is relatively straightforward, and involves computing weakest preconditions, and checking non-emptiness of intersection of polyhedral sets. For computing continuous successors of an abstract state $A$, we use a strategy inspired by the techniques used in CHEckMATE and d/dt. However, while tools such as d/dt are designed to compute a "good" approximation of the continuous successors of $A$, we are interested in checking if this set intersects with a new abstract state permitting many optimizations. Postulating the verification problem for hybrid systems as a search problem in the abstract system has many benefits compared to the traditional approach of computing approximations of reachable sets, and our experiments indicate significant improvements in time and space requirements compared to a tool such as d/dt.

The success of our scheme crucially depends on the choice of the predicates used for abstraction. In this paper, we focus on identifying such predicates automatically by analyzing spurious counter-examples generated by the search in the abstract state-space. Counter-example guided refinement of abstractions has been used in multiple contexts before, for instance, to identify the relevant timing constraints in verification of timed automata [5], to identify the relevant boolean predicates in verification of $\mathrm{C}$ programs [7], and to identify the relevant variables in symbolic model checking [11]. We present the basic techniques for analyzing counter-examples, techniques for discovering new predicates that will rule out spurious counter-examples, optimizations of these techniques, implementation of these in our verifier, and case studies demonstrating the promise of the approach. Counter-example guided refinement of abstractions for hybrid systems is being independently explored by the hybrid systems group at CMU [10].

The abstract counter-example consists of a sequence of abstract states leading from an initial state to a state violating the property. The analysis problem is to check if the corresponding sequence can be traversed in the concrete system. We perform a forward search from the initial abstract state following the given counter-example. The analysis relies on techniques for polyhedral approximations of the reachable sets under continuous dynamics. We also implemented a local test that checks for feasibility of pairwise transitions, and this proves to be effective in many cases. If the counter-example is found to be infeasible, then we wish to identify new predicates that would rule out this sequence in the refined abstract space. This reduces to the problem of finding predicates that separate two sets of polyhedra. We present a greedy strategy for identifying such predicates. After discovering new predicates, we include these to the set of predicates 
used before, and rerun the search in the refined abstract state-space. We demonstrate the feasibility using three case studies. The first one involves verification of a parametric version of Fischer's protocol. The second one involves analysis of a thermostat model, and the third analyzes a model of an adaptive cruise controller. In each of these cases, we show how counter-example analysis can be effective in discovering the predicates that are needed for establishing safety.

\section{Predicate Abstraction for Linear Hybrid Systems}

In this section, we briefly recap the definitions of predicate abstraction for linear hybrid systems and the search strategy in the abstract space as outlined in [3].

\subsection{Mathematical Model}

We denote the set of all $n$-dimensional linear expressions $l: \mathbb{R}^{n} \rightarrow \mathbb{R}$ with $\Sigma_{n}$ and the set of all $n$-dimensional linear predicates $\pi: \mathbb{R}^{n} \rightarrow \mathbb{B}$, where $\mathbb{B}:=\{0,1\}$, with $\mathcal{L}_{n}$. A linear predicate is of the form $\pi(x):=\sum_{i=1}^{n} a_{i} x_{i}+a_{n+1} \sim 0$, where $\sim \in\{\geq,>\}$ and $\forall i \in\{1, \ldots, n+1\}: a_{i} \in \mathbb{R}$. The set of finite sets of $n$-dimensional linear predicates is denoted by $\mathcal{C}_{n}$, where an element of $\mathcal{C}_{n}$ represents the conjunction of its elements.

Definition 1 (Linear Hybrid System). An n-dimensional linear hybrid system is a tuple $H=\left(\mathcal{X}, L, X_{0}, I, f, T\right)$ with the following components:

$-\mathcal{X} \subseteq \mathbb{R}^{n}$ is a convex polyhedron representing the continuous state-space.

$-L$ is a finite set of locations. The state-space of $H$ is $X=L \times \mathcal{X}$. Each state thus has the form $(l, x)$, where $l \in L$ is the discrete part of the state, and $x \in \mathcal{X}$ is the continuous part.

- $X_{0} \subseteq X$ is the set of initial states. It is assumed that for all locations $l \in L$, the set $\left\{x \in \mathcal{X} \mid(l, x) \in X_{0}\right\}$ is a convex polyhedron.

$-I: L \rightarrow \mathcal{C}_{n}$ assigns to each location $l \in L$ a finite set of linear predicates $I(l)$ defining the invariant conditions that constrain the value of the continuous part of the state while the discrete location is $l$. The hybrid automaton can only stay in location $l$ as long as the continuous part of the state $x$ satisfies $I(l)$, i.e. $\forall \pi \in I(l): \pi(x)=1$. We write $\mathcal{I}_{l}$ for the invariant set of location $l$, that is the set of all points $x$ satisfying all predicates in $I(l)$.

- $f: L \rightarrow\left(\mathbb{R}^{n} \rightarrow \mathbb{R}^{n}\right)$ assigns to each location $l \in L$ a continuous vector field $f(l)$ on $x$. While at location $l$ the evolution of the continuous variable is governed by the differential equation $\dot{x}=f(l)(x)$. We restrict our attention to hybrid automata with linear continuous dynamics, that is, for every location $l \in L$, the vector field $f(l)$ is linear, i.e. $f(l)(x)=A_{l} x$ where $A_{l}$ is an $n \times n$ matrix. The analysis can also be applied to systems having linear continuous dynamics with uncertain, bounded input of the form $\dot{x}=A_{l} x+B_{l} u$.

$-T \subseteq L \times L \times \mathcal{C}_{n} \times\left(\Sigma_{n}\right)^{n}$ is a relation capturing discrete transition jumps between two discrete locations. A transition $\left(l, l^{\prime}, g, r\right) \in T$ consists of an initial location $l$, a destination location $l^{\prime}$, a set of guard constraints $g$ and 
a linear reset mapping $r$. From a state $(l, x)$ where all predicates in $g$ are satisfied the hybrid automaton can jump to location $l^{\prime}$ at which the continuous variable $x$ is reset to a new value $r(x)$. We write $\mathcal{G}_{t} \subseteq \mathcal{I}_{l}$ for the guard set of a transition $t=\left(l, l^{\prime}, g, r\right) \in T$ which is the set of points satisfying all linear predicates of $g$ and the invariant of the location $l$.

\subsection{Transition System Semantics}

We define the semantics of a hybrid automaton by formalizing its underlying transition system. For simplicity we consider the system $\dot{x}=A_{l} x$, and we denote the flow of this system with $\Phi_{l}(x, t)=e^{A_{l} t} x$. The underlying transition system of $H$ is $T_{H}=\left\{X, \rightarrow, X_{0}\right\}$. The state-space of the transition system is the statespace of $H$, i.e. $X=L \times \mathcal{X}$. The transition relation $\rightarrow \subseteq X \times X$ between states of the transition system is defined as the union of two relations $\rightarrow_{C}, \rightarrow_{D} \subseteq X \times X$. The relation $\rightarrow_{C}$ describes transitions due to continuous flows, whereas $\rightarrow_{D}$ describes transitions due to discrete jumps.

$$
\begin{gathered}
(l, x) \rightarrow_{C}(l, y) \text { iff } \exists t \in \mathbb{R}_{\geq 0}: \Phi_{l}(x, t)=y \wedge \forall t^{\prime} \in[0, t]: \Phi_{l}\left(x, t^{\prime}\right) \in \mathcal{I}_{l} . \\
(l, x) \rightarrow_{D}\left(l^{\prime}, y\right) \text { iff } \exists\left(l, l^{\prime}, g, r\right) \in T: x \in \mathcal{G}_{t} \wedge y=r(x) \wedge y \in \mathcal{I}_{l^{\prime}} .
\end{gathered}
$$

\subsection{Discrete Abstraction}

We define a discrete abstraction of the hybrid system $H=\left(\mathcal{X}, L, X_{0}, I, f, T\right)$ with respect to a given $k$-dimensional vector of $n$-dimensional linear predicates $\Pi=\left(\pi_{1}, \pi_{2}, \ldots, \pi_{k}\right) \in\left(\mathcal{L}_{n}\right)^{k}$. We can partition the continuous state-space $\mathcal{X} \subseteq$ $\mathbb{R}^{n}$ into at most $2^{k}$ states, corresponding to the $2^{k}$ possible boolean evaluations of $\Pi$; hence, the infinite state-space $X$ of $H$ is reduced to $|L| 2^{k}$ states in the abstract system. From now on, we refer to the hybrid system $H$ as the concrete system and its state-space $X$ as the concrete state-space.

Definition 2 (Abstract state-space). Given an $n$-dimensional hybrid system $H=\left(\mathcal{X}, L, X_{0}, f, I, T\right)$ and a $k$-dimensional vector $\Pi \in\left(\mathcal{L}_{n}\right)^{k}$ of $n$-dimensional linear predicates we can define an abstract state as a tuple $(l, \boldsymbol{b})$, where $l \in$ $L$ and $\boldsymbol{b} \in \mathbb{B}^{k}$. The abstract state-space for a $k$-dimensional vector of linear predicates hence is $Q_{\Pi}:=L \times \mathbb{B}^{k}$. We define a concretization function $C_{\Pi}$ : $\mathbb{B}^{k} \rightarrow 2^{\mathcal{X}}$ for a vector of linear predicates $\Pi=\left(\pi_{1}, \ldots, \pi_{k}\right) \in\left(\mathcal{L}_{n}\right)^{k}$ as $C_{\Pi}(\boldsymbol{b}):=$ $\left\{x \in \mathcal{X} \mid \forall i \in\{1, \ldots, k\}: \pi_{i}(x)=b_{i}\right\}$.

Definition 3 (Discrete Abstraction). Given a hybrid system $H=\left(\mathcal{X}, L, X_{0}\right.$, $f, I, T)$, we define its abstract system with respect to a vector of linear predicates $\Pi$ as the transition system $H_{\Pi}=\left(Q_{\Pi}, \stackrel{\Pi}{\rightarrow}, Q_{0}\right)$ where

- the set of initial states is $Q_{0}=\left\{(l, \boldsymbol{b}) \in Q_{\Pi} \mid \exists x \in C_{\Pi}(\boldsymbol{b}):(l, x) \in X_{0}\right\}$;

- the abstract transition relation $\stackrel{\Pi}{\rightarrow} \subseteq Q_{\Pi} \times Q_{\Pi}$ is defined as the union of the following two relations $\stackrel{\Pi}{\rightarrow} D, \stackrel{\Pi}{\rightarrow}_{C} \subseteq Q_{\Pi} \times Q_{\Pi}$. The relation $\stackrel{\Pi}{\rightarrow} D$ represents 
transitions in the abstract state-space due to discrete jumps, whereas $\stackrel{\Pi}{\rightarrow} C$ represents transitions due to continuous flows:

$$
\begin{aligned}
& (l, \boldsymbol{b}) \stackrel{\Pi}{\rightarrow}_{D}\left(l^{\prime}, \boldsymbol{b}^{\prime}\right) \quad \text { iff } \exists\left(l, l^{\prime}, g, r\right) \in T, x \in C_{\Pi}(\boldsymbol{b}) \cap \mathcal{G}_{t}: \\
& (l, x) \rightarrow_{D}\left(l^{\prime}, r(x)\right) \wedge r(x) \in C_{\Pi}\left(\boldsymbol{b}^{\prime}\right) ; \\
& (l, \boldsymbol{b}) \stackrel{\Pi}{\rightarrow}_{C}\left(l, \boldsymbol{b}^{\prime}\right) \quad \text { iff } \exists x \in C_{\Pi}(\boldsymbol{b}), t \in \mathbb{R}_{\geq 0}: \Phi_{l}(x, t) \in C_{\Pi}\left(\boldsymbol{b}^{\prime}\right) \wedge \\
& \forall t^{\prime} \in[0, t]: \Phi_{l}\left(x, t^{\prime}\right) \in \mathcal{I}_{l} \text {. }
\end{aligned}
$$

\subsection{Searching the Abstract State-Space}

Given a hybrid system $H$ we want to verify certain safety properties. We define a property by specifying a set of unsafe locations $U \subseteq L$ and a set $\mathcal{B} \subseteq \mathcal{X}$ of unsafe continuous states. The property is said to hold for the hybrid system $H$ iff there is no valid trace that leads to some state in $\mathcal{B}$ while in an unsafe location. We implemented an on-the-fly search of the abstract state-space giving priority to computing discrete successors rather than continuous successors, as this is generally much faster. Computing discrete successors is relatively straightforward, and involves computing weakest preconditions, and checking non-emptiness of intersection of polyhedral sets. For computing continuous successors of an abstract state $A$, we compute the polyhedral slices of states reachable at fixed times $r, 2 r, 3 r, \ldots$ for a suitably chosen $r$, and then, take convex-hull of all these polyhedra to over-approximate the set of all states reachable from $A$. We are only interested in checking if this set intersects with a new abstract state. This approach has many benefits compared to the traditional approach of computing approximations of reachable sets, one of them being the fact that the expensive operation of computing continuous successors is applied only to abstract states, and not to intermediate polyhedra of unpredictable shapes and complexities.

We include an optimization technique in the search strategy. For each concrete counter-example in the concrete hybrid system, there exists an equivalent counter-example that has the additional constraint that there are no two consecutive transitions due to continuous flow. This is due to the additivity of flows of hybrid systems, namely $(l, x) \rightarrow_{C}\left(l, x^{\prime}\right) \wedge\left(l, x^{\prime}\right) \rightarrow_{C}\left(l, x^{\prime \prime}\right) \Rightarrow(l, x) \rightarrow_{C}\left(l, x^{\prime \prime}\right)$. We are hence searching only for counter-examples in the abstract system that do not have two consecutive transitions due to continuous flow.

\section{Counter-Example Analysis}

An abstract counter-example consists of a sequence of abstract states and transitions leading from an initial state to a state violating the property. The analysis problem is to check if the corresponding sequence of modes and discrete switches can be traversed in the concrete system. The analysis relies on techniques for polyhedral approximations of the reachable sets under continuous dynamics. To speed up the feasibility analysis, we also implemented a local test that checks for feasibility of pairwise transitions, and this often proves to be effective. 


\subsection{Global Analysis Algorithm}

We denote the set of transition labels $\Sigma_{T}$ as $\Sigma_{T}=T \cup\{C\}$, denoting that either a discrete transition or a continuous transition occurred. For the subsequent definitions of counter-examples in the abstract state-space we use the following notation for transitions due to discrete jumps for $t=\left(l, l^{\prime}, g, r\right) \in T$ :

$$
(l, \boldsymbol{b}) \stackrel{\Pi}{\rightarrow}_{t}\left(l^{\prime}, \boldsymbol{b}^{\prime}\right) \text { iff } \exists x \in C_{\Pi}(\boldsymbol{b}) \cap \mathcal{G}_{t}:(l, x) \rightarrow_{D}\left(l^{\prime}, r(x)\right) \wedge r(x) \in C_{\Pi}\left(\boldsymbol{b}^{\prime}\right) .
$$

Definition 4. An abstract path $p$ in the abstract state-space given by the vector of predicates $\Pi$ of length $n \geq 0$ is a pair $(\boldsymbol{a}, \boldsymbol{t}) \in\left(Q_{\Pi}\right)^{n+1} \times\left(\Sigma_{T}\right)^{n}$, such that $\boldsymbol{a}=\left(a_{0}, \ldots, a_{n}\right)$ and $\boldsymbol{t}=\left(t_{0}, \ldots, t_{n-1}\right)$ with $t_{i} \in \Sigma_{T}, a_{0}=\left(l_{0}, \boldsymbol{b}_{\mathbf{0}}\right) \in Q_{0}$, and $\forall 0 \leq i \leq n-1: a_{i} \stackrel{\Pi}{\rightarrow} t_{i} a_{i+1}$. The set of abstract paths of length $n$ given by the vector of predicates $\Pi$ is denoted by $\mathcal{P}_{n}^{\Pi}$. A counter-example is an abstract path $p=(\boldsymbol{a}, \boldsymbol{t})=\left(\left(a_{0}, \ldots, a_{n}\right),\left(t_{0}, \ldots, t_{n-1}\right)\right)$, such that $a_{n}$ is a violation of the property to be proven. We call the sequence of abstract states $\boldsymbol{a}=\left(a_{0}, \ldots, a_{n}\right)$ of a counter-example $p=(\boldsymbol{a}, \boldsymbol{t})$ an unlabeled counter-example.

The counter-example analysis problem is twofold. The first objective is to check whether a counter-example in the abstract system corresponds to a counter-example in the concrete system. In case that the analysis finds that this particular counter-example cannot be traversed in the concrete system, we want the analysis to identify one or more new predicates that would rule out closely related counter-examples in the refined abstract state-space. The refined abstract state-space is defined by adding these predicates to the previous set of predicates used in the abstract state-space search. We define the notion of refinement between abstract paths to formalize the concept of closely related abstract paths.

Definition 5. A vector of predicates $\Pi^{\prime} \in\left(\mathcal{L}_{n}\right)^{k^{\prime}}$ refines a vector of predicates $\Pi \in\left(\mathcal{L}_{n}\right)^{k}$, if its corresponding set of predicates includes all predicates in $\Pi$.

Definition 6. An abstract state $a^{\prime}=\left(l^{\prime}, \boldsymbol{b}^{\prime}\right) \in Q_{\Pi^{\prime}}$ for the vector of predicates $\Pi^{\prime}$ refines another abstract state $a=(l, \boldsymbol{b}) \in Q_{\Pi}$ for the vector of predicates $\Pi$, iff $l=l^{\prime}$ and $C_{\Pi^{\prime}}\left(\boldsymbol{b}^{\prime}\right) \subseteq C_{\Pi}(\boldsymbol{b})$.

Definition 7. An abstract path $p^{\prime}=\left(\left(a_{0}^{\prime}, \ldots, a_{n}^{\prime}\right),\left(t_{0}^{\prime}, \ldots, t_{n-1}^{\prime}\right)\right) \in \mathcal{P}_{n}^{\Pi^{\prime}}$ for $a$ vector of predicates $\Pi^{\prime}$ refines another abstract path $p=\left(\left(a_{0}, \ldots, a_{n}\right),\left(t_{0}, \ldots\right.\right.$, $\left.\left.t_{n-1}\right)\right) \in \mathcal{P}_{n}^{\Pi}$ for a vector of predicates $\Pi$, with $a_{i}=\left(l_{i}, \boldsymbol{b}_{\boldsymbol{i}}\right)$ and $a_{i}^{\prime}=\left(l_{i}^{\prime}, \boldsymbol{b}_{\boldsymbol{i}}^{\prime}\right)$, iff $\Pi^{\prime}$ refines $\Pi, \forall 0 \leq i \leq n$ : $a_{i}^{\prime}$ refines $a_{i}$, and $\forall 0 \leq i \leq n-1: t_{i}^{\prime}=t_{i}$.

During the counter-example analysis we define Pre : $Q_{\Pi} \times \Sigma_{T} \times Q_{\Pi} \rightarrow 2^{\mathcal{X}}$ and Post : $2^{\mathcal{X}} \times \Sigma_{T} \times Q_{\Pi} \rightarrow 2^{\mathcal{X}}$ functions that consider only the abstract states or the concretely reachable state space rather than the whole continuous statespace $\mathcal{X}$. The computation of these takes into consideration the concretization of the abstract state, as well as the invariants and guards of the system. We define the functions Pre and Post with $a=(l, \boldsymbol{b})$ and $a^{\prime}=\left(l^{\prime}, \boldsymbol{b}^{\prime}\right)$ as: 


$$
\begin{aligned}
& \operatorname{Pre}\left(a, t, a^{\prime}\right)=\left\{\begin{array}{r}
\left\{\begin{array}{c}
x \in C_{\Pi}(\boldsymbol{b}) \cap \mathcal{G}_{t} \mid \\
r(x) \in C_{\Pi}\left(\boldsymbol{b}^{\prime}\right) \cap \mathcal{I}_{l^{\prime}}
\end{array}\right\} \quad: \quad t=\left(l, l^{\prime}, g, r\right) \\
\left\{\begin{array}{c}
x \in C_{\Pi}(\boldsymbol{b}) \cap \mathcal{I}_{l} \mid \exists \tau \in \mathbb{R}_{\geq 0} \\
\Phi_{l}(x, \tau) \in C_{\Pi}\left(\boldsymbol{b}^{\prime}\right) \cap \mathcal{I}_{l^{\prime}} \\
\wedge \forall \tau^{\prime} \in[0, \tau]: \Phi_{l}\left(x, \tau^{\prime}\right) \in \mathcal{I}_{l^{\prime}}
\end{array}\right\}
\end{array} \quad: \quad t=C\right. \\
& \operatorname{Post}\left(P, t, a^{\prime}\right)=\left\{\begin{array}{c}
\operatorname{Post}\left(\left\{\begin{array}{c}
x \in C_{\Pi}\left(\boldsymbol{b}^{\prime}\right) \cap \mathcal{I}_{l^{\prime}} \mid \\
\exists y \in \mathcal{G}_{t} \cap P: x=r(y)
\end{array}\right\}, C, a^{\prime}\right) \quad: \quad t=\left(l, l^{\prime}, g, r\right) ; \\
\left\{\begin{array}{c}
x \in C_{\Pi}\left(\boldsymbol{b}^{\prime}\right) \cap \mathcal{I}_{l^{\prime}} \mid \exists \tau \in \mathbb{R}_{\geq 0} \\
\exists y \in P: \Phi_{l^{\prime}}(y, \tau)=x \\
\wedge \forall \tau^{\prime} \in[0, \tau]: \Phi_{l^{\prime}}\left(y, \tau^{\prime}\right) \in \mathcal{I}_{l^{\prime}}
\end{array}\right\}: t=C .
\end{array}\right.
\end{aligned}
$$

Our counter-example analysis algorithm is presented in algorithm 1 . The set $R_{0}$ is the part of the initial state-space that is covered by the abstract state $\left(l, \boldsymbol{b}_{\mathbf{0}}\right)$. We then compute the concretely reachable state-space of each abstract state of the counter-example. For each $1 \leq i \leq n$ we compute $R_{i}$ as the reachable region after $i$ transitions according to the counter-example. It is hence clear that if $R_{i}=\emptyset$ for some $i$ then the counter-example is spurious.

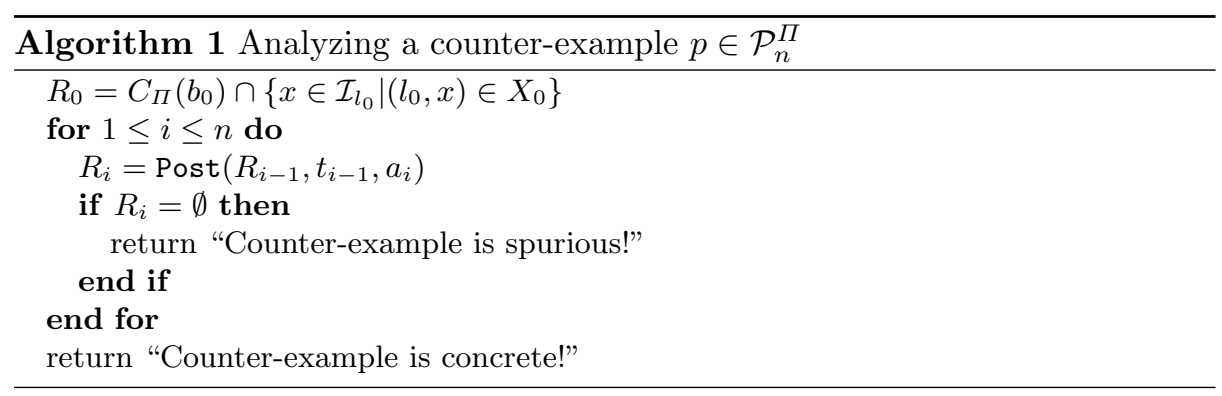

In the case that we found that the counter-example is spurious, we want to use the counter-example to find new predicates. Consider a counter-example $p \in \mathcal{P}_{n}^{\Pi}$, such that $R_{k+1}=\emptyset$ and $R_{k} \neq \emptyset$ for $0 \leq k<n$. We call $t_{k}$ the failing transition of the counter-example $p$. Then we can prove the following lemma 1

Lemma 1. Given a counter-example $p=(\boldsymbol{a}, \boldsymbol{t})=\left(\left(a_{0}, \ldots, a_{n}\right),\left(t_{0}, \ldots, t_{n-1}\right)\right)$ $\in \mathcal{P}_{n}^{\Pi}$ where $t_{k}$ is the failing transition, we have $R_{k} \cap \operatorname{Pre}\left(a_{k}, t_{k}, a_{k+1}\right)=\emptyset$.

We want to add new predicates to the vector $\Pi$, so that the refined vector $\Pi^{\prime}$ does not allow a refined (unlabeled) counter-example of $p$ to reappear. Consider a strategy that adds predicates to the set $\Pi$ that correspond to a separation of $R_{k}$ from $\operatorname{Pre}\left(a_{k}, t_{k}, a_{k+1}\right)$ for a failing transition $t_{k}$. This means that we are looking for a refined set of predicates $\Pi^{\prime}$ of $\Pi$, such that every refined abstract

\footnotetext{
${ }^{1}$ Please note, that we omit all the proofs in this paper for the sake of brevity.
} 
state intersects at most with one of the two sets $R_{k}$ and $\operatorname{Pre}\left(a_{k}, t_{k}, a_{k+1}\right)$. We define the notion of separation in terms of polyhedral sets, since we approximate the set of reachable states by polyhedral slices in the implementation of the tool. It should be noted here that we use under-approximations of the reachable sets of states during the analysis of counter-examples while we over-approximated the reachable sets of states during the search in the abstract state-space.

Definition 8 (Separating predicates). Let $\mathcal{P}=\left\{P_{1}, \ldots, P_{n}\right\}$ and $\mathcal{Q}=$ $\left\{Q_{1}, \ldots, Q_{m}\right\}$ be two disjoint sets of convex polyhedra. We denote by $\bigcup \mathcal{P}$ and $\bigcup \mathcal{Q}$ the union of all polyhedra in $\mathcal{P}$ and $\mathcal{Q}$. A finite vector of linear predicates $\Pi=\left(\pi_{1}, \pi_{2}, \ldots, \pi_{k}\right)$ separates $\mathcal{P}$ and $\mathcal{Q}$ iff for all $\boldsymbol{b} \in \mathbb{B}^{k}$, at least one of the two sets $\left(C_{\Pi}(\boldsymbol{b}) \cap \bigcup \mathcal{P}\right)$ and $\left(C_{\Pi}(\boldsymbol{b}) \cap \bigcup \mathcal{Q}\right)$ is empty.

The predicates in $\Pi$ are called separating predicates. Note that such a vector $\Pi$ always exist 2 , but it is often not unique.

Theorem 1. Assume a counter-example $p \in \mathcal{P}_{n}^{\Pi}$ for a vector of predicates $\Pi$ such that $t_{k}$ is the failing transition. If $\Pi^{\prime}$ refines $\Pi$ and additionally contains predicates corresponding to a separation of $R_{k}$ from $\operatorname{Pre}\left(a_{k}, t_{k}, a_{k+1}\right)$, and we find a refined counter-example $p^{\prime} \in \mathcal{P}_{n}^{\Pi^{\prime}}$ of $p$, then there exists a failing transition $t_{j}$ in $p^{\prime}$, such that $j<k$.

As a single counter-example $p$ is of finite length, the above theorem assures us that after a finite number of iterations, a refinement of $p$ will not be possible.

\subsection{Locally Infeasible Abstract States}

In this section we present a second counter-example analysis algorithm, which checks a counter-example quickly for a common cause of spurious counter-examples. We also show that this analysis produces new predicates with stronger implications for subsequent searches in the refined abstract state-space.

Definition 9. For a path $p=(\boldsymbol{a}, \boldsymbol{t}) \in \mathcal{P}_{n+1}^{\Pi}$ given the vector of predicates $\Pi$, with $\boldsymbol{a}=\left(a_{0}, \ldots, a_{n+1}\right)=\left(\left(l_{0}, \boldsymbol{b}_{\mathbf{0}}\right), \ldots,\left(l_{n+1}, \boldsymbol{b}_{\boldsymbol{n}+\mathbf{1}}\right)\right)$ and $\boldsymbol{t}=\left(t_{0}, \ldots, t_{n}\right)$, we say that an abstract state $a_{i}$ for $1 \leq i \leq n$ is locally infeasible, iff

$$
\operatorname{Post}\left(C_{\Pi}\left(\boldsymbol{b}_{\boldsymbol{i}-\mathbf{1}}\right), t_{i-1}, a_{i}\right) \cap \operatorname{Pre}\left(a_{i}, t_{i}, a_{i+1}\right)=\emptyset \text {. }
$$

The detection of locally infeasible abstract states can be implemented in a straight-forward fashion. In addition, we can easily compute new predicates that disallow refined counter-examples. If a state $a_{i}$ is locally infeasible, then we can use the fact that our implemented optimization technique guarantees that either $t_{i-1}$ or $t_{i}$ is a discrete transition. If $t_{i-1}$ is discrete, one reasonable choice is to use the predicates corresponding to the constraints of the polyhedral sets representing Post $\left(C_{\Pi}\left(\boldsymbol{b}_{\boldsymbol{i}-\mathbf{1}}\right), t_{i-1}, a_{i}\right)$ in the refined search. Otherwise, a possible approach is to use the predicates corresponding to $\operatorname{Pre}\left(a_{i}, t_{i}, a_{i+1}\right)$ in the refined search. We denote this strategy of picking new predicates by LocalStrategy.

\footnotetext{
${ }^{2}$ It is easy to see that we can simply take the linear constraints of all polyhedra from $P$ or from $Q$ to determine $\Pi$.
} 
We can now prove the following two theorems about using the strategy LocalStrategy in case we find a locally infeasible abstract state. The theorems formalize that by using this strategy we can assure that a refinement of the (unlabeled) counter-example will not be found in subsequent searches.

Theorem 2. Assume a counter-example $p \in \mathcal{P}_{n}^{\Pi}$ for a vector of predicates $\Pi$, such that there is a locally infeasible abstract state $a_{i}$ in $p$. A search in the refined abstract state-space given by the strategy LocalStrategy to find new predicates will not find a counter-example that is a refinement of $p$.

Theorem 3. Assume a hybrid system $H=\left(\mathcal{X}, L, X_{0}, I, f, T\right)$ with the properties that $\forall l \in L:\left(l, l^{\prime}, g, r\right) \in T \Rightarrow l \neq l^{\prime}$, and $\forall\left(l, l^{\prime}, g_{1}, r_{1}\right),\left(l, l^{\prime}, g_{2}, r_{2}\right) \in T$ : $g_{1}=g_{2} \wedge r_{1}=r_{2}$. Additionally assume a counter-example $p=(\boldsymbol{a}, \boldsymbol{t}) \in \mathcal{P}_{n}^{\Pi}$ for a vector of predicates $\Pi$, such that there is a locally infeasible abstract state $a_{i}$ in $p$. Then a search in the refined abstract state-space obtained using the strategy LocalStrategy to refine the set of predicates $\Pi$ will not produce a refined unlabeled counter-example.

\section{Computing Separating Predicates}

In the previous section we described two counter-example analysis algorithms. If the counter-example is found to be infeasible, then we wish to identify one or more new predicates that would rule out this sequence in the refined abstract space. This reduces to the problem of finding one or more predicates that separate two sets of polyhedra. We present a greedy strategy for identifying the separating predicates. After discovering new predicates, we then include these predicates to the set of predicates used before, and rerun the search in the refined abstract state-space defined by the enriched predicate set.

\subsection{Separating Two Disjoint Convex Polyhedra}

Let $P$ and $Q$ be two disjoint convex polyhedra. To separate them, we define the distance between $P$ and $Q$ as follows: $d(P, Q)=\inf \{d(p-q) \mid p \in P \wedge q \in Q\}$ where $d(\cdot)$ denotes the Euclidean distance. Since $P$ and $Q$ are disjoint, $d(P, Q)$ is positive. Let $p^{*} \in P$ and $q^{*} \in Q$ be points that form a pair of closest points. We denote by $s\left(p^{*}, q^{*}\right)$ the line segment with extreme points $p^{*}$ and $q^{*}$. The half-space $\mathcal{H}$ which is normal to $s\left(p^{*}, q^{*}\right)$ and has $q^{*}$ as a supporting point can be written as: $\mathcal{H}=\left\{x \mid\left\langle p^{*}-q^{*}, x\right\rangle \geq\left\langle p^{*}-q^{*}, q^{*}\right\rangle\right\}$. We denote by $\overline{\mathcal{H}}$ the complement of $\mathcal{H}$.

Lemma 2. The polyhedron $Q$ is contained in $\mathcal{H}$ and $P$ is contained in $\overline{\mathcal{H}}$.

We remark that Lemma 2 also holds for any half-space which is normal to $s\left(p^{*}, q^{*}\right)$ and passes through an arbitrary point in $s\left(p^{*}, q^{*}\right)$. Hence, any such half-space can be used to define a separating predicate. To compute $d(P, Q)$ as well as $p^{*}$ and $q^{*}$, there exist efficient algorithms [8] which take time $\mathcal{O}\left(K_{P}+K_{Q}\right)$ where $K_{P}$ and $K_{Q}$ are the number of vertices of $P$ and $Q$. 


\subsection{Separating Two Disjoint Sets of Convex Polyhedra}

We proceed with the problem of finding a set $\Pi$ of separating predicates for two sets of convex polyhedra $\mathcal{P}_{1}$ and $\mathcal{P}_{2}$. In order to keep the size of the abstract state space as small as possible, we want to find $\Pi$ with the smallest number of predicates. Many related polyhedral separation problems have been considered in literature 1718 . However, the solutions proposed in these works are only for two and three dimensional polyhedra. On the other hand, even in low dimensions most separation problems were shown to be intractably hard. In three dimensions the problem of finding a minimum facet-separator for two polyhedral solids is NP-complete [15]. Our objective is not to find an optimal solution but to develop methods which are effective on the problem of separating reachable sets of hybrid systems for abstraction refinement purposes.
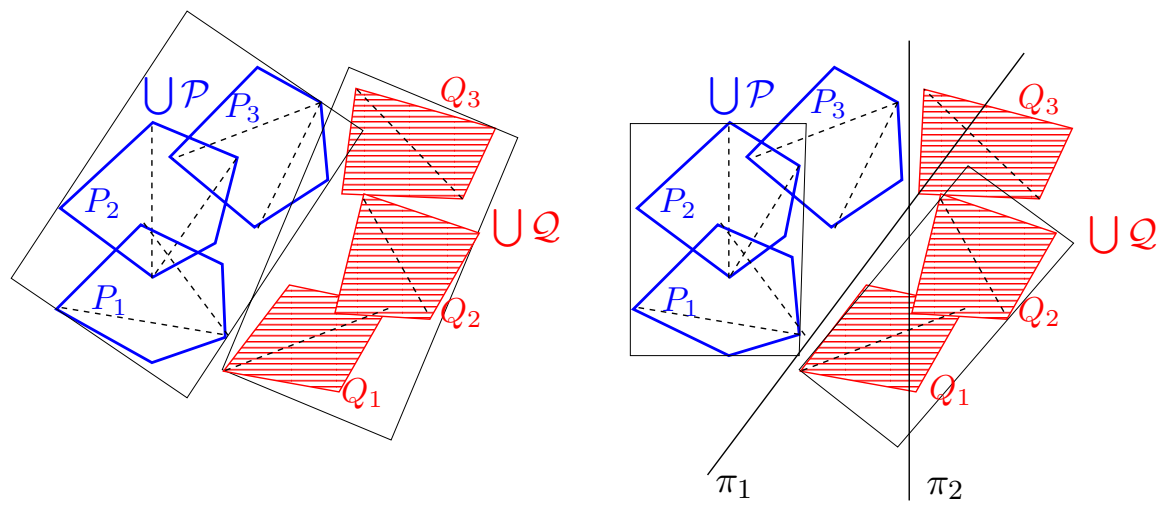

Fig. 1. Subdividing the sets $\mathcal{P}=\left\{P_{1}, P_{2}, P_{3}\right\}$ and $\mathcal{Q}=\left\{Q_{1}, Q_{2}, Q_{3}\right\}$ respectively into $\left\{P_{1}, P_{2}\right\},\left\{P_{3}\right\}$ and $\left\{Q_{1}, Q_{2}\right\},\left\{Q_{3}\right\}$ allows to find two separating predicates $\pi_{1}$ and $\pi_{2}$.

Our solution is based on the following observation. Given two set of polyhedra $\mathcal{P}_{1}$ and $\mathcal{P}_{2}$, if the convex hulls of $\mathcal{P}_{1}$ and $\mathcal{P}_{2}$ are disjoint, then one can apply the method presented in the previous section to find a separating predicate. If the convex hulls intersect, it is clear that $\mathcal{P}_{1}$ and $\mathcal{P}_{2}$ cannot be separated by a single hyperplane. The main idea is to divide $\mathcal{P}_{1}$ and $\mathcal{P}_{2}$ into subsets of polyhedra such that their convex hulls do not intersect allowing to find a separating predicate. The procedure of subdivision can be done in a hierarchical way. We begin with all polyhedra in $\mathcal{P}_{1}$ and $\mathcal{P}_{2}$ and recursively subdivide until the convex hulls are pairwise disjoint. Moreover, for efficiency purposes, instead of convex hulls, we can use approximations by non-axis-aligned bounding boxes which are easier to compute and test for overlaps (see figure 1). One way to compute tight fitting bounding boxes is to align the axes of the box in the directions along which the vertices of the polyhedra tend to lie. From the vertices of the polyhedra we can 
determine the matrix of covariance and take its largest eigenvectors to define the orientation of the box.

Our method for computing separating predicates is summarized in algorithm 2. We denote by $\mathcal{H}(\pi)$ the half-space defined by predicate $\pi$. Given a set $\mathcal{P}$ of polyhedra, $\operatorname{chull}(\mathcal{P})$ and $\operatorname{bbox}(\mathcal{P})$ are respectively the convex hull and the nonaxis-aligned bounding box of $\mathcal{P}$. The set $\mathcal{S}(\mathcal{P}, \pi)=\{s \in \mathcal{P} \mid s \subseteq \mathcal{H}(\pi)\}$ is the largest subset of $\mathcal{P}$ lying entirely inside $\mathcal{H}(\pi)$, and $\operatorname{Int}(\mathcal{P}, \pi)=\{s \cap \mathcal{H}(\pi) \mid s \in$ $\mathcal{P} \wedge s \cap \mathcal{H}(\pi) \neq \emptyset\}$ is the intersection of $\bigcup \mathcal{P}$ with $\mathcal{H}(\pi)$. The core of the algorithm is a procedure, called sep, which computes a separating predicate for two disjoint polyhedra using the method presented in section 4.1. Two sets of polyhedra $\mathcal{P}_{1}$ and $\mathcal{P}_{2}$ are called separable if $\operatorname{conv}\left\{\mathcal{P}_{1}\right\} \cap \operatorname{conv}\left\{\mathcal{P}_{2}\right\}=\emptyset$ where conv is a convex-approximation operation which can be chull or bbox. The notation $\operatorname{separable}\left(\mathcal{P}_{1}, \mathcal{P}_{2}\right)$ in the algorithm indicates that $\mathcal{P}_{1}$ and $\mathcal{P}_{2}$ are separable.

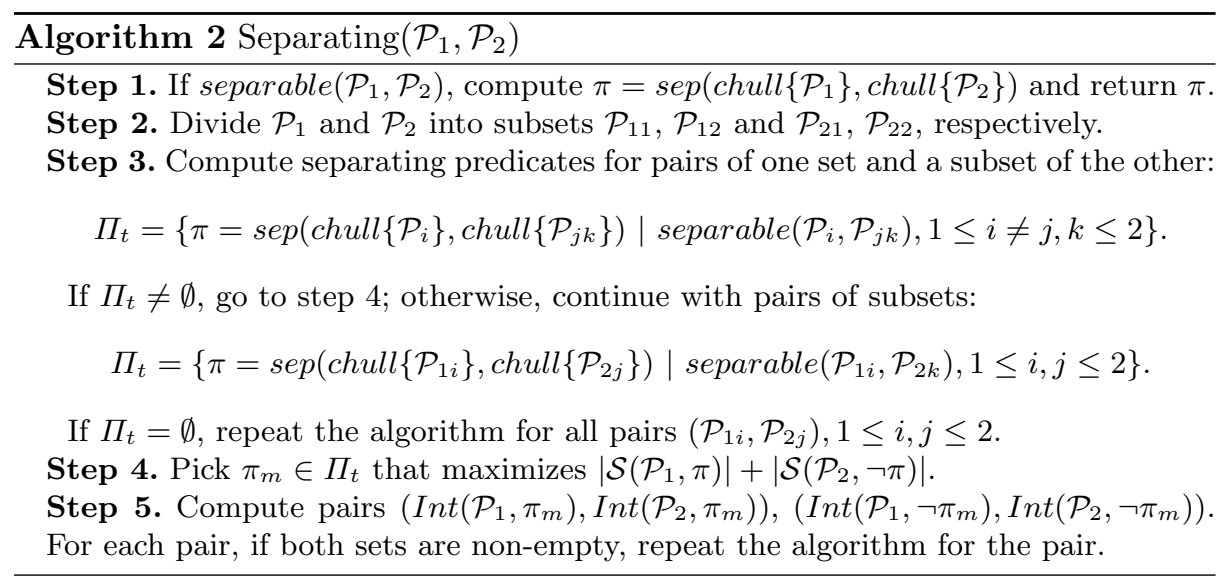

We briefly sketch the proof of the correctness of algorithm 2. As one can see from step 4, we use a greedy strategy to choose separating predicates, that is we select the one that can separate the largest number of polyhedra. The goal of step 5 is to exclude the subsets of $\bigcup \mathcal{P}_{1}$ and $\bigcup \mathcal{P}_{2}$ that the selected predicate $\pi_{m}$ can separate. Indeed, if one of the sets $\operatorname{Int}\left(\mathcal{P}_{1}, \pi_{m}\right)$ and $\operatorname{Int}\left(\mathcal{P}_{2}, \pi_{m}\right)$ is empty, then either $\mathcal{P}_{1}$ or $\mathcal{P}_{2}$ lies entirely outside the half-space $\mathcal{H}\left(\pi_{m}\right)$. This means that the predicate $\pi_{m}$ can separate a part of one set from the other, and we only need to continue with the remaining part.

One factor that determines the number of separating predicates is the subdivision in step 2 . The way we subdivide the sets $\mathcal{P}_{1}$ with view of avoiding interference of the resulting subsets with $\mathcal{P}_{2}$ is as follows. We first try to split $\mathcal{P}_{1}$ into two subsets such that one contains all the polyhedra entirely outside $\operatorname{conv}\left(\mathcal{P}_{2}\right)$. If this subset is empty, then we split $\mathcal{P}_{1}$ with respect to a hyperplane which is perpendicular to the longest side of $\operatorname{bbox}\left(\mathcal{P}_{1}\right)$ and passes through its 
centroid. Another option for the normal of the splitting hyperplane is the line passing through the two most distant points.

We now briefly discuss the bound on the number of predicates algorithm 2 can produce. It is easy to see that the upper bound corresponds to the case where no splitting can produce separable subsets, which requires to consider all pairs of polyhedra. In other words, in this case, each time algorithm 2 finds a separating predicate $\pi_{m}, \mid\left(\operatorname{Int}\left(\mathcal{P}_{1}, \pi_{m}\right)|=| \mathcal{P}_{1} \mid-1\right.$ and $\mid\left(\operatorname{Int}\left(\mathcal{P}_{2}, \pi_{m}\right)|=| \mathcal{P}_{2} \mid\right.$; similarly, $\mid\left(\operatorname{Int}\left(\mathcal{P}_{1}, \neg \pi_{m}\right)|=| \mathcal{P}_{1} \mid\right.$ and $\mid\left(\operatorname{Int}\left(\mathcal{P}_{2}, \neg \pi_{m}\right)|=| \mathcal{P}_{2} \mid-1\right.$. This means that in each side of the half-space of $\pi_{m}$, two sets of polyhedra remain to be separated and the size of one set is decreased by 1 . For example, if $\left|\mathcal{P}_{1}\right|=$ $\left|\mathcal{P}_{2}\right|=K$, we can prove that algorithm 2 produces in worst case $2^{K+1}-1$ predicates. It is important to note that this worst case typically happens when the polyhedra in each set are all disjoint and intertwine with those in the other set. However, in this context, reachable sets to be separated are often connected. Hence, in many practical cases, convex hulls or non-axis-aligned bounding boxes are relatively good approximations and the number of separating predicates produced by algorithm 2 is often much smaller than this bound. Finally, we can use the following lemma to achieve better efficiency.

Lemma 3. If a set of predicates $\Pi$ separates the boundaries of $\mathcal{P}_{1}$ and $\mathcal{P}_{2}$ then it separates $\mathcal{P}_{1}$ and $\mathcal{P}_{2}$.

To prove the lemma, we remark that $\Pi$ separates $\mathcal{P}_{1}$ and $\mathcal{P}_{2}$ iff any line segment between a point in $\mathcal{P}_{1}$ and another point in $\mathcal{P}_{2}$ intersects with the hyperplane of at least one predicates in $\Pi$. Hence if $\Pi$ separates the boundaries of $\mathcal{P}_{1}$ and $\mathcal{P}_{2}$ then it separates $\mathcal{P}_{1}$ and $\mathcal{P}_{2}$ since any line segment connecting points in the interior of two disjoint sets must cross the boundaries of both sets. Using lemma 3 we can consider only some boundary layer of $\mathcal{P}_{1}$ and $\mathcal{P}_{2}$, which allows to obtain tighter convex approximations and thus reduces splitting.

\section{Implementation and Experimentation}

We presented foundations for automated verification of safety properties of hybrid systems by combining the ideas of counter-example guided predicate abstraction and polyhedral approximation of reachable sets of linear continuous dynamics. The presented counter-example analysis tool extends previous work on predicate abstraction of hybrid systems 3. Our current prototype implementation of the predicate abstraction model checking and the counter-example analysis tool are both implemented in $\mathrm{C}++$ using library functions of the hybrid systems reachability tool d/dt 6 . We implemented a translation procedure from CHARon 2] source code to the predicate abstraction input language which is based on the $d / d t$ input language. Our tool uses the polyhedral libraries CDD and QHull. We have implemented the global analysis algorithm, the local feasibility check, as well as the computation of separating predicates as part of the counter-example analysis tool. 


\subsection{Fischer's Mutual Exclusion}

We first look at an example of mutual exclusion which uses time-based synchronization in a multi-process system. We want to implement a protocol that allows a shared resource to be used exclusively by at most one of two processes at any given time. The state machines for the two processes are shown in figure 2 The example is small enough to be used effectively for an illustration of our approach.

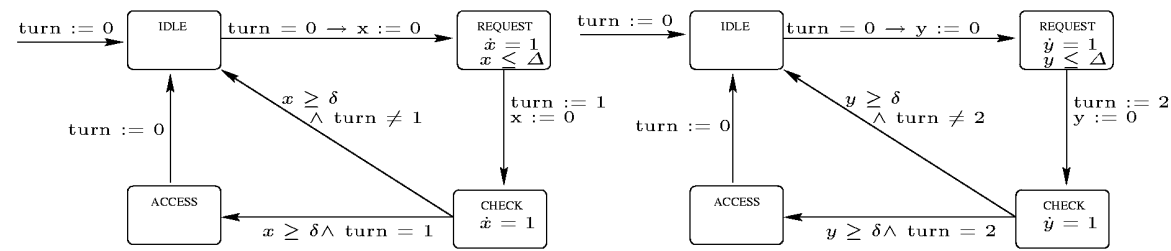

Fig. 2. The two processes for the mutual exclusion example

The possible execution traces depend on the two positive parameters $\Delta$ and $\delta$. If the parameters are such that $\Delta \geq \delta$ is true, we can find a counter-example that proves the two processes may access the shared resource at the same time. On the other hand, if $\delta>\Delta$, then the system preserves mutual exclusive use of the shared resource. We use this example to illustrate the use of the local feasibility check of counter-examples for the case that $\delta>\Delta$. Consider the abstract system defined by the predicates used in the description of the 2-process Fischer's mutual exclusion protocol. These are: $x \geq \delta, y \geq \delta, x \leq \Delta, y \leq \Delta, \delta>$ $\Delta, \Delta>0, \delta>0, x \geq 0$ and $y \geq 0$. The search in the abstract state-space finds a counter-example of length nine. The third abstract state $a_{3}$ in the counterexample has both processes in their respective Request locations, turn $=0$, and $0 \leq x \leq \Delta, 0 \leq y \leq \Delta$. The following state $a_{4}$ can be reached by a discrete transition $t_{d}$, and the first process is now in its Check location, while turn $=1$ and $0 \leq x \leq \Delta, 0 \leq y \leq \Delta$. The fifth abstract state $a_{5}$ can then be reached by a continuous transition $t_{c}$, so that the locations and the turn variable are unchanged, but now we have $x>\delta, 0 \leq y \leq \Delta$. Then, $a_{4}$ is locally infeasible, as shown by projection onto the variables $x$ and $y$ :

$$
\begin{gathered}
\text { Post }_{\mid(x, y)}\left(a_{3}, t_{d}, a_{4}\right)=\left\{(x, y)^{T} \in \mathbb{R}^{2} \mid 0 \leq x \leq y \leq \Delta<\delta\right\} \text { and } \\
\operatorname{Pre}_{\mid(x, y)}\left(a_{4}, t_{c}, a_{5}\right)=\left\{(x, y)^{T} \in \mathbb{R}^{2} \mid 0 \leq y<x \leq \Delta<\delta\right\} ;
\end{gathered}
$$

hence, we know that $\operatorname{Post}\left(a_{3}, t_{d}, a_{4}\right) \cap \operatorname{Pre}\left(a_{4}, t_{c}, a_{5}\right)=\emptyset$. Following the strategy LocalStrategy we include the only one new predicate $x \leq y$ to the set of predicates. In the next iteration with this refinement of the abstract statespace, we obtain a symmetrical locally infeasible counter-example. The strategy LocalStrategy then suggests the symmetric predicate $y \leq x$. The subsequent reachability analysis finds 54 reachable abstract states in the refined abstract state-space, which all maintain the mutual exclusion property. 


\subsection{Thermostat}

We have also successfully applied our counter-example guided predicate abstraction technique to verify a thermostat example. We present this case study to illustrate the global counter-example analysis algorithm as well as the procedure to separate two disjoint sets of polyhedra. The single hybrid automaton in this case study is shown in figure 3 It contains a timer clock and a temperature Temp. The initial location is Heat and the initial set is clock $=0,5 \leq$ Temp $\leq 10$, and the bad set is Temp $<4.5$ in any location. The model can be proven correctly by adding the predicate clock $\leq 0$ to the predicates that are already mentioned in the model as transition guards and location invariants. In this case, the abstraction uses ten predicates and finds 35 reachable abstract states.

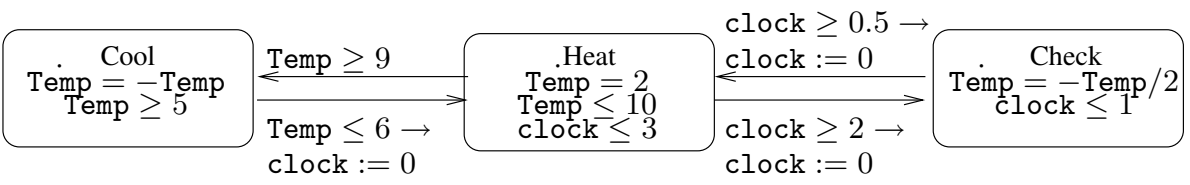

Fig. 3. The Thermostat example. We omit the differential equation clock $=1$ in all three locations.

For purposes of illustration, we start the verification using our counterexample guided predicate abstraction toolkit with the predicates mentioned in the model; that means, we are searching for a set of predicates that can be used to refine this initial set to be able to prove the safety of the given model. Note that picking the aforementioned predicate clock $\leq 0$ would suffice. In addition, to illustrate the global analysis algorithm and the separating routine only, we skip the local feasibility checking algorithm. The first iteration of our algorithm produces a spurious counter-example of length 7 after 11 abstract states have been discovered by the search of the abstract state-space. The separation routine suggests the following four linear predicate to refine the abstract state-space:

$$
\begin{aligned}
& 0.979265 * \mathrm{Temp}+0.202584 * \text { clock }<=9.34423 \\
& 0.872555 * \mathrm{Temp}+0.488515 * \text { clock }<=8.16961 \\
& 0.428587 * \text { Temp }+0.9035 * \text { clock }<=4.11184 \\
& -0.0680518 * \text { Temp }+0.997682 * \text { clock }<=-0.439659
\end{aligned}
$$

Please notice the last suggested predicate and its similarity to the predicate mentioned before. The model designer may have been able to use this suggested set of predicates to refine the abstract state space by adding the predicate clock $\leq 0$.

Following our example, after refining the predicates with the help of these four predicates, the system still finds a spurious counter-example, and suggest four more predicates. In a third round after discovering another spurious counter-example, the system generates eleven more predicates, one of which is $0.0139043 *$ Temp $+0.999903 *$ clock $<=0.152558$. The total set of 28 predi- 
cates is in the following iteration enough to prove the thermostat example safe. The search in the abstract state-space finds 358 reachable abstract states.

\subsection{Coordinated Adaptive Cruise Control}

We have also successfully applied our predicate abstraction technique to verify a model of the Coordinated Adaptive Cruise Control mode of a vehicle-tovehicle coordination system. This case study is provided by the PATH project (see http: //www-path.eecs.berkeley.edu). We first briefly describe the model omitting a more detailed discussion for the sake of brevity. The goal of this mode is to maintain the car at some desired speed $v_{d}$ while avoiding collision with a car in front. Let $x$ and $v$ denote the position and velocity of the car. Let $x_{l}, v_{l}$ and $a_{l}$ denote respectively the position, velocity and acceleration of the car in front. Since we want to prove that no collision happens regardless of the behavior of the car in front, this car is treated as disturbance, more precisely, the derivative of its acceleration is modeled as uncertain input ranging in $\left[d a_{l \min }, d a_{l \max }\right]$.

The closed-loop system can be modeled as a hybrid automaton with 5 continuous variables and 8 locations. The invariants of the locations and the transition guards are specified by the operation regions and switching conditions of the controller together with the bounds on the speed and acceleration. In order to prove that the controller can guarantee that no collision between the cars can happen, we specify an unsafe set as $x_{l}-x \leq 0$ in all locations. To define initial predicates, in addition to the constraints of the invariants and guards, we use the predicate of the bad set allowing to distinguish safe and unsafe states and predicates representing the initial set. Assuming that the follower car is faster than the preceding car, and a too small initial separation of the two cars, the tool finds a counter-example that corresponds to a real trace in the concrete system. On the other hand, if the two cars start with a large enough initial separation, the combined verification approach enabled us to prove safety of the abstract system which implies safety of the concrete system.

\section{References}

1. R. Alur, C. Courcoubetis, N. Halbwachs, T.A. Henzinger, P. Ho, X. Nicollin, A. Olivero, J. Sifakis, and S. Yovine. The algorithmic analysis of hybrid systems. Theoretical Computer Science, 138:3-34, 1995.

2. R. Alur, T. Dang, J. Esposito, Y. Hur, F. Ivančić, V. Kumar, I. Lee, P. Mishra, G. Pappas, and O. Sokolsky. Hierarchical modeling and analysis of embedded systems. Proceedings of the IEEE, 91(1), January 2003.

3. R. Alur, T. Dang, and F. Ivančić. Reachability analysis of hybrid systems via predicate abstraction. In Hybrid Systems: Computation and Control, Fifth International Workshop, LNCS 2289. Springer-Verlag, 2002.

4. R. Alur and D.L. Dill. A theory of timed automata. Theoretical Computer Science, 126:183-235, 1994.

5. R. Alur, A. Itai, R.P. Kurshan, and M. Yannakakis. Timing verification by successive approximation. Information and Computation, 118(1):142-157, 1995. 
6. E. Asarin, O. Bournez, T. Dang, and O. Maler. Approximate reachability analysis of piecewise-linear dynamical systems. In Hybrid Systems: Computation and Control, Third International Workshop, LNCS 1790, pages 21-31. 2000.

7. T. Ball and S. Rajamani. Bebop: A symbolic model checker for boolean programs. In SPIN 2000 Workshop on Model Checking of Software, LNCS 1885. 2000.

8. S. Cameron. A comparison of two fast algorithms for computing the distance between convex polyhedra. IEEE Transactions on Robotics and Automation, 13(6):915-920, 1997.

9. A. Chutinan and B.K. Krogh. Verification of polyhedral-invariant hybrid automata using polygonal flow pipe approximations. In Hybrid Systems: Computation and Control, Second International Workshop, LNCS 1569, pages 76-90. 1999.

10. E. Clarke, A. Fehnker, Z. Han, B. Krogh, O. Stursberg, and M. Theobald. Verification of hybrid systems based on counterexample-guided abstraction refinement. In Tools and Algorithms for the Construction and Analysis of Systems, 2003.

11. E. Clarke, O. Grumberg, S. Jha, Y. Lu, and H. Veith. Counterexample-guided abstraction refinement. In Computer Aided Verification, pages 154-169, 2000.

12. E.M. Clarke and R.P. Kurshan. Computer-aided verification. IEEE Spectrum, 33(6):61-67, 1996.

13. J.C. Corbett, M.B. Dwyer, J. Hatcliff, S. Laubach, C.S. Pasareanu, Robby, and H. Zheng. Bandera: Extracting finite-state models from Java source code. In Proceedings of 22nd International Conference on Software Engineering. 2000.

14. P. Cousot and R. Cousot. Abstract interpretation: a unified lattice model for static analysis of programs by construction or approximation of fixpoints. In Proceedings of the 4th ACM Symposium on Principles of Programming Languages, 1977.

15. G. Das and D. Joseph. The complexity of minimum convex nested polyhedra. In Canadian Conference on Computational Geometry, 1990.

16. C. Daws, A. Olivero, S. Tripakis, and S. Yovine. The tool Kronos. In Hybrid Systems III: Verification and Control, LNCS 1066. Springer-Verlag, 1996.

17. D. Dobkin and D. Kirkpatrick. Determining the separation of preprocessed polyhedra - a unified approach. In Proc. of ICALP'90, pages 400-413, 1990.

18. H. Edelsbrunner and F.P. Preparata. Minimum polygon separation. Information and Computation, 77:218-232, 1987.

19. T.A. Henzinger, P. Ho, and H. Wong-Toi. НүTесн: the next generation. In Proceedings of the 16th IEEE Real-Time Systems Symposium, pages 56-65, 1995.

20. G.J. Holzmann. The model checker SPIN. IEEE Transactions on Software Engineering, 23(5):279-295, 1997.

21. G.J. Holzmann and M.H. Smith. Automating software feature verification. Bell Labs Technical Journal, 5(2):72-87, 2000.

22. K. Larsen, P. Pettersson, and W. Yi. UPPAAL in a nutshell. Springer International Journal of Software Tools for Technology Transfer, 1, 1997.

23. C. Loiseaux, S. Graf, J. Sifakis, A. Bouajjani, and S. Bensalem. Property preserving abstractions for the verification of concurrent systems. Formal Methods in System Design Volume 6, Issue 1, 1995. 